Research Paper

\title{
Two Closely Related Human Members of Chitinase-like Family, CHI3LI and CHI3L2, Activate ERK1/2 in 293 and U373 Cells but Have the Difierent In- fluence on Cell Proliieration
}

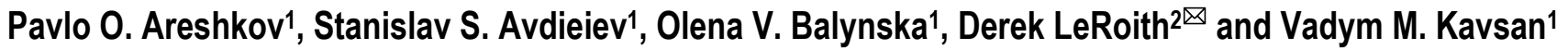 \\ 1. Department of Biosynthesis of Nucleic Acids, Institute of Molecular Biology and Genetics, 150 Zabolotnogo street, Kyiv 03680, Ukraine. \\ 2. Division of Endocrinology, Metabolism, and Bone Diseases, Mount Sinai School of Medicine, One Gustave L. Levy Place, Box 1055, New \\ York, NY 10029, USA.
}

Corresponding author: Derek LeRoith, the Division of Endocrinology, Metabolism, and Bone Diseases, Mount Sinai School of Medicine (e-mail: derek.leroith@mssm.edu).

(C) Ivyspring International Publisher. This is an open-access article distributed under the terms of the Creative Commons License (http:/ / creativecommons.org/ licenses/by-nc-nd/3.0/). Reproduction is permitted for personal, noncommercial use, provided that the article is in whole, unmodified, and properly cited.

Received: 2011.06.17; Accepted: 2011.08.21; Published: 2011.11.16

\begin{abstract}
The activation of extracellular signal-regulated kinases (ERKI/2) has been associated with specific outcomes. Sustained activation of ERKI/2 by nerve growth factor (NGF) is associated with translocation of ERKs to the nucleus of $\mathrm{PCI} 2$ cells and precedes their differentiation into sympathetic-like neurons whereas transient activation by epidermal growth factor (EGF) leads to cell proliferation. It was demonstrated that different growth factors initiating the same cellular signaling pathways may lead to the different cell destiny, either to proliferation or to the inhibition of mitogenesis and apoptosis. Thus, further investigation on kinetic differences in activation of certain signal cascades in different cell types by biologically different agents are necessary for understanding the mechanisms as to how cells make a choice between proliferation and differentiation.

It was reported that chitinase 3-like I (CHI3LI) protein promotes the growth of human synovial cells as well as skin and fetal lung fibroblasts similarly to insulin-like growth factor I (IGFI). Both are involved in mediating the mitogenic response through the signal-regulated kinases ERKI/2. In addition, CHI3LI which is highly expressed in different tumors including glioblastomas possesses oncogenic properties. As we found earlier, chitinase 3-like 2 (CHI3L2) most closely related to human CHI3LI also showed increased expression in glial tumors at both the RNA and protein levels and stimulated the activation of the MAPK pathway through phosphorylation of ERKI/2 in 293 and U87 MG cells. The work described here demonstrates the influence of $\mathrm{CHI} 3 \mathrm{~L} 2$ and $\mathrm{CHI} \mathrm{LL}$ on the duration of MAPK cellular signaling and phosphorylated ERKI/2 translocation to the nucleus. In contrast to the activation of ERKI/2 phosphorylation by CHI3LI that leads to a proliferative signal (similar to the EGF effect in PCI 2 cells), activation of ERKI/2 phosphorylation by CHI3L2 (similar to NGF) inhibits cell mitogenesis and proliferation.
\end{abstract}

Key words: chitinase 3-like 1 (CHI3L1, HC gp-39, YKL-40) protein; chitinase 3-like 2 (CHI3L2, YKL-39) protein; ERK1/2; MAP kinase).

\section{Introduction}

Investigation of the molecular mechanisms of proliferation and differentiation as an answer to cel- lular stimulation by certain growth factors led to the creation of a general concept, as to how the cell itself 
makes a choice regarding further development. The reaction of the cell to definitive stimuli is mediated by signal events, which are specific to the cell and to the localization of necessary transcription factors. Further investigations on kinetic differences in activation of certain signal cascades by different bio-active agents are necessary for the understanding of the mechanisms that determine the destiny of different cells.

Determination of adequate cell response to the specific stimuli is thought to be dependent on the duration of ERK1/2 activity [1]. NGF stimulation of PC12 cells gave the sustained activation together with the nuclear translocation of ERK1/2, which led to the neuronal differentiation, while EGF gave short activation of ERK1/2 without change in cellular localization, resulted in a proliferative signal [2]. Plenty of ligands provoked significantly similar set of expressed immediate-early genes in PC12 cells and fibroblasts [3], however, resultant cell phenotype may be very distinct.

Mammalian genomes code a set of homologous CLPs regardless the absence of chitin (poly-N-glucosamine) synthesis in mammalian species. According to classification based on amino acid sequence similarities, CLPs have been grouped in a family 18 glycosyl hydrolases [4]. In humans there are six proteins of this family [5] and CHI3L1 is the most highly investigated protein of the group. It has a molecular weight of about $40 \mathrm{kDa}$ and $\mathrm{N}$-glycosylation at Asn $^{60}$. CHI3L1 monomer consists of 383 amino acid residues, contains a signal peptide $\mathrm{Met}^{1}-\mathrm{Ala}^{21}$ for secretion and two structural domains. The expression of the CHI3L1 gene was found in synovial cells and articular cartilage chondrocytes. It is increased significantly in various tumors, and cell lines derived from such tumors, including tumors of the bone, brain, breast, lung, and ovary [6]. Increased CHI3L1 gene expression was found in glioblastoma and may be a survival marker [7-10]. It was shown recently, that CHI3L1 protects against apoptosis [11] and possesses oncogenic properties [12].

CHI3L1 addition to human synovial cells as well as to skin and fetal lung fibroblasts activated MAPK and PI3K signaling pathways which were involved in mediating the mitogenic response. Dose-dependent growth stimulation occurred in a concentration range similar to the well-studied mitogen IGF1. Selective inhibition of the MAPK and AKT signaling pathways indicates that both are necessary for mitogenic effect of CHI3L1. Thus, CHI3L1 may have growth factor properties for the connective-tissue cells [13].

Chitinase 3-like 2 (CHI3L2) is closely related to CHI3L1 and has significant sequence homology (49-53\%) with other mammalian CLPs. A clue to its function was gained from the structural similarity with CHI3L1 in size, nucleotide and amino acid sequences ( $47 \%$ and $51 \%$ homology, correspondingly), they have the same $\mathrm{NH}_{2}$-terminal amino acid residues as noted in their alternative names (YKL-40 and YKL-39). CHI3L2 was originaly isolated from the cultured medium of primary human articular cartilage chondrocytes as a protein that co-purified with CHI3L1 [14]. Previously, we revealed an increased expression of both, CHI3L1 [7, 9] and CHI3L2 [15] genes in glioblastoma. However, Western blot analysis did not show simultaneous production of CHI3L1 and CHI3L2, apparently indicating their different functions. As we have found recently, CHI3L2 similarly to CHI3L1 also activated ERK1/2 phosphorylation in cells of tumor and non-tumor origin [16].

The work described here demonstrates the role of CHI3L1 and CHI3L2 in the control of mitogenesis and proliferation as well as the influence of CHI3L1 and CHI3L2 on the duration of MAPK cellular signaling and phosphorylated ERK1/2 nuclear translocation in 293 cells and U373 cells as a comparison to that observed for EGF or NGF in PC12 cells.

\section{Materials and Methods}

\section{Cell cultures}

293 cells (Human Embryonic Kidney 293 cells, also often referred to HEK 293, or less precisely as HEK cells) and U373 cells (Human glioblastoma-astrocytoma, epithelial-like cell line) kindly provided by Prof. I. Gout (UCL, Cell Signaling and Metabolic Regulation Dept., UK) were grown in DMEM supplemented with $10 \%$ FBS and $100 \mu \mathrm{g} / \mathrm{ml}$ penicillin/100 units/ml streptomycin in an environment of 95\% air/5\% $\mathrm{CO}_{2}$.

\section{Assessment of mitogenic activity}

Mitogenic activity was assessed by determination of DNA-synthesis rates of treated and untreated cultures. 293 cells or U373 cells were seeded into 24-well tissue-culture plates and allowed to grow to near-confluence in DMEM supplemented with 10\% FBS, followed by a $24 \mathrm{hrs}$ serum-starvation period in unsupplemented DMEM. CHI3L1, CHI3L2 or FBS were then added in DMEM at the concentrations indicated, followed by $\left[{ }^{3} \mathrm{H}\right]$ thymidine $(3 \mu \mathrm{Ci} / \mathrm{ml}) 2 \mathrm{hrs}$ later. Cultures were terminated after $24 \mathrm{hrs}$ of exposure and the cell layers were briefly washed twice by PBS and lysed in $0.5 \mathrm{M} \mathrm{NaOH}, 0.5 \%$ SDS. DNA was collected on glass-fiber filters and washed with 5\% trichloroacetic acid. $\left[{ }^{3} \mathrm{H}\right]$ thymidine content was determined by liquid scintillation spectroscopy using a Perkin Elmer scintillation counter. 


\section{Cell Proliferation Assay}

293 cells and U373 cells were treated as for the assessment of mitogenic activity described above or exposed to the ERK1/ 2 inhibitor U0126 $(20 \mu \mathrm{M})$ for 30 min and then stimulated with $100 \mathrm{ng} / \mathrm{ml} \mathrm{CHI3L1} \mathrm{or}$ CHI3L2 for $60 \mathrm{~min}$. Cellular proliferation was estimated using the CellTiter 96 AQueous One Solution cell proliferation assay kit (Promega, Madison, WI). 20 $\mathrm{ml}$ of 2-(4',5'-dimethyl-2'-thiazolyl)-3-(4'-sulfophenyl) (MTS) solution was added to each well. The plate was kept for $4 \mathrm{hrs}$ in a $\mathrm{CO}_{2}$ incubator, and the absorbance at $490 \mathrm{~nm}$ was recorded with 96 -well plate reader. The background readings in the wells with medium were subtracted from the sample well read outs.

\section{Apoptosis assay}

293 cells and U373 cells were treated as for the assessment of mitogenic activity described above. For activated caspase-3/7 analysis, cells were incubated for $1 \mathrm{hr}$ with reconstituted Caspase 3/7-Glo reagent (Promega) and then, the luminescence signal generated after cleavage of DEVD-aminoluciferin substrate by caspase $3 / 7$, was measured using 96 -well plate reader.

\section{Proteins and antibodies}

Native CHI3L1 purified from supernatant of conditioned MG-63 cell (Human osteosarcoma cell line) medium [17] was kindly provided by Dr. A. Iershov (Department of Biosynthesis of Nucleic Acids, Institute of Molecular Biology and Genetics, Kyiv, Ukraine). Synthesis of recombinant CHI3L2 was performed according to our previous description [15]. Protein products were analyzed by $12 \%$ SDS-PAGE and Coomassie Brilliant Blue R250 staining. Purified $\mathrm{CHI} 3 \mathrm{~L} 1$ and $\mathrm{CHI} 3 \mathrm{~L} 2$ were stored frozen at $-20^{\circ} \mathrm{C}$ in aliquots to avoid repeated freeze-thawing and denaturation.

Antibodies were obtained from multiple sources and used at the specified dilutions for immunofluorescence microscopy: p44/42 MAP (Erk1/2) (L34F12) Mouse mAb (Cell Signaling Technology, Danvers, MA) 1:100 (anti-phospho-ERK); ERK1 (K-23) Rabbit polyclonal IgG (Santa Cruz Biotechnology, Santa Cruz, CA) 1:100 (pan-anti-ERK); Fluorescein Anti-Mouse IgG $(\mathrm{H}+\mathrm{L})$ as well as Texas $\operatorname{Red}{ }^{\circledR}$ Anti-Rabbit IgG (Vector Laboratories Inc., Burlingame, CA) 1:400; and for immunoblots: pERK (E-4) Mouse monoclonal IgG (Santa Cruz) 1:2000 (anti-phospho-ERK); ERK1 (K-23) Rabbit polyclonal IgG 1:3000 (pan-anti-ERK); Anti-Mouse IgG (H+L), HRP Conjugate and Anti-Rabbit IgG $(\mathrm{H}+\mathrm{L})$, HRP Conjugate (Promega) 1:20000 (HRP-anti-mouse IgG), (HRP-anti-rabbit IgG). The reagents for enhanced chemiluminescence (ECL) from Sigma-Aldrich Co. and Fluka (Buchs, Switzerland) were used for the visualization of immunoreactive bands on Western blots. The MAP kinase kinase (MEK) inhibitor U0126 was obtained from Cayman Chemical (Ann Arbor, MI).

\section{Analysis of ERK I/2 activation}

For investigation of ERK1/2 phosphorylation 293 cells or U373 cells were seeded into 6-well tissue culture plates in $10 \%$ FBS contained DMEM and allowed to grow to near-confluence. Cells were serum-starved for $24 \mathrm{hrs}$ or exposed to the ERK1/2 inhibitor U0126, as described above followed by addition of $100 \mathrm{ng} / \mathrm{ml} \mathrm{CHI3L} 1$ or CHI3L2 for time periods up to $2 \mathrm{hrs}$. At the end of the incubation periods, cell layers were washed twice in ice-cold PBS and whole cell lysates were mixed with $2 \times$ Laemmli sample buffer, boiled, proteins resolved by $10 \%$ SDS-PAGE and transferred to nitrocellulose membrane. Membranes were blocked for $1 \mathrm{hr}$ at room temperature with $5 \%$ powdered skim milk in TBS with $0.05 \%$ Triton X-100 (TBST), reacted with anti-phospho-ERK at $4^{\circ} \mathrm{C}$ overnight, and then incubated with HRP-anti-mouse IgG for $1 \mathrm{hr}$. Blots were developed with an ECL detection system. Membranes were incubated in stripping buffer $(0.5 \mathrm{M} \mathrm{NaCl}, 0.2 \mathrm{M}$ Gly, $\mathrm{pH} 2.5)$ for $15 \mathrm{~min}$ at $70^{\circ} \mathrm{C}$, and then they were washed twice in TBST, blocked, and incubated with pan-anti-ERK for $1 \mathrm{hr}$ at room temperature. After incubation with HRP-anti-rabbit IgG for $1 \mathrm{hr}$, total ERK1/2 was detected with ECL. Specific activation of MAP kinases was measured by densitometric analysis of Western-blot signals using Scion Image 1.62c program (NIH ImageJ; NIH, Bethesda, MD).

\section{Immunofluorescence and confocal microscopy}

293 cells were seeded on coverslips and allowed to grow to near-confluence. Cells were then serum-starved for $24 \mathrm{hrs}$, followed by exposure to CHI3L1 or CHI3L2 at $100 \mathrm{ng} / \mathrm{ml}$ for time period up to 2 hrs. After treatment, the cells were washed in cold PBS, fixed with $10 \%$ paraformaldehyde and permeabilized in $-20^{\circ} \mathrm{C}$ methanol, washed three times for 5 min each with PBS and blocked with blocking buffer (2\% BSA in PBS) for $30 \mathrm{~min}$ at room temperature. Incubations were performed at room temperature with antibodies diluted in blocking buffer. Slides were mounted using PVA-DABCO (Fluka) and images were captured with a Zeiss LSM 510 Meta confocal microscope (Germany). All images represent a single confocal section, taken from the bottom surface of the cells being analyzed. 


\section{Statistical analysis}

Comparisons between two groups were done by using the independent samples $\mathbf{t}$ test. A value of $\mathrm{P}<0.05$ was considered statistically significant.

\section{Results}

\section{Antagonistic effect of CHI3LI and CHI3L2 on 293 cell and $U 373$ cell growth}

The influence of CHI3L2 protein application on the mitogenesis and growth of 293 cells and U373 cells, was evaluated by incorporation of $\left[{ }^{3} \mathrm{H}\right]$ thymidine into cellular DNA and proliferative activity using the MTS test. Unexpectedly, in contrast to CHI3L1, a dose-dependent decrease of $\left[{ }^{3} \mathrm{H}\right]$ thymidine incorporation (Figs. 1A, B) and cell proliferation (Figs. 1C, D) was observed in the cells

293 cells

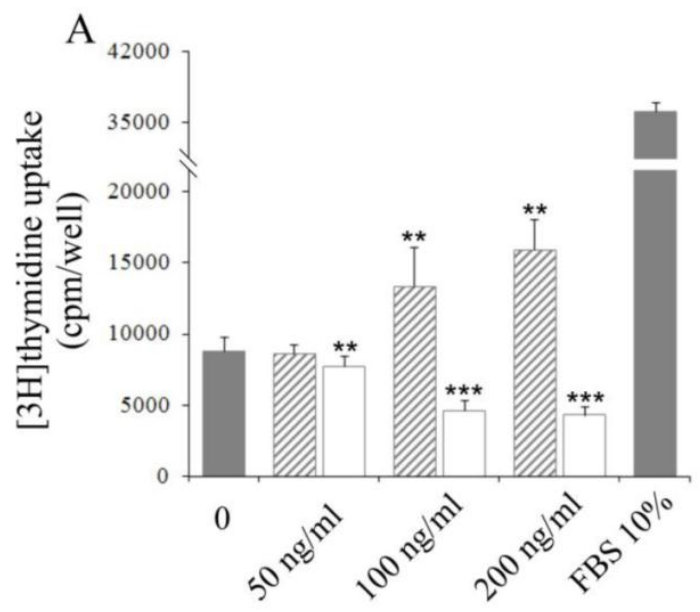

U373 cells

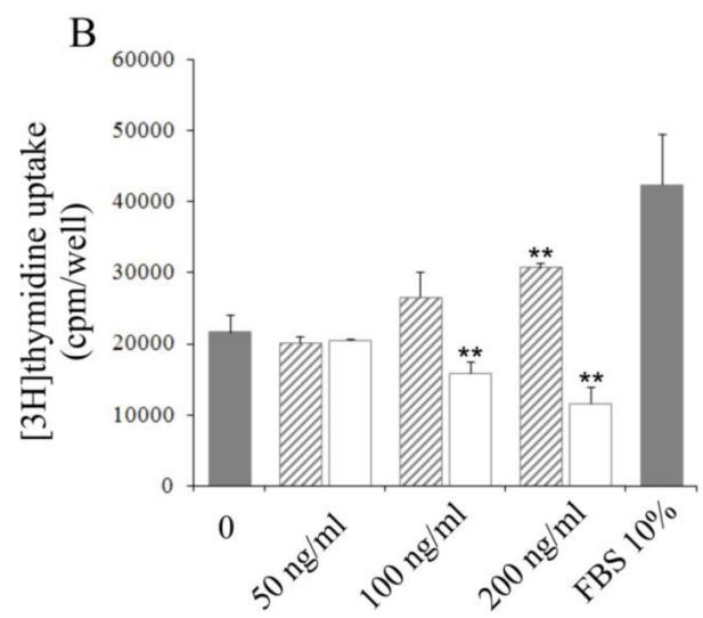

treated with CHI3L2 at concentrations of 50, 100 and $200 \mathrm{ng} / \mathrm{ml}$. The magnitude of the response decreased by $8,5 \pm 16 \%$ fold (for 293 cells) or $3,7 \pm 38 \%$ fold (for U373 cells) in $\left[{ }^{3} \mathrm{H}\right]$ thymidine incorporation and $3,9 \pm 48 \%$ (for 293 cells) or $4,3 \pm 61 \%$ (for U373 cells) in proliferation compared to that elicited by $10 \%$ FBS and CHI3L2 $(200 \mathrm{ng} / \mathrm{ml})$. It was decreased by $2 \pm 24 \%$ fold (for 293 cells) or $1,7 \pm 32 \%$ fold (for U373 cells) in $\left[{ }^{3} \mathrm{H}\right]$ thymidine incorporation and $2,1 \pm 50 \%$ in proliferation for both cell types compared to that elicited by untreated cells and cells treated with CHI3L2 (200 $\mathrm{ng} / \mathrm{ml})$. The simultaneous addition of both chitinase-like proteins showed the inhibition of $\left[{ }^{3} \mathrm{H}\right]$ thymidine incorporation in 293 cells and U373 cells stimulated with CHI3L1 addition alone (Figs. 2A, $\mathrm{B})$, and vice versa, the apoptotic influence of CHI3L2 was inhibited with CHI3L1 addition (Figs. 2C, D).
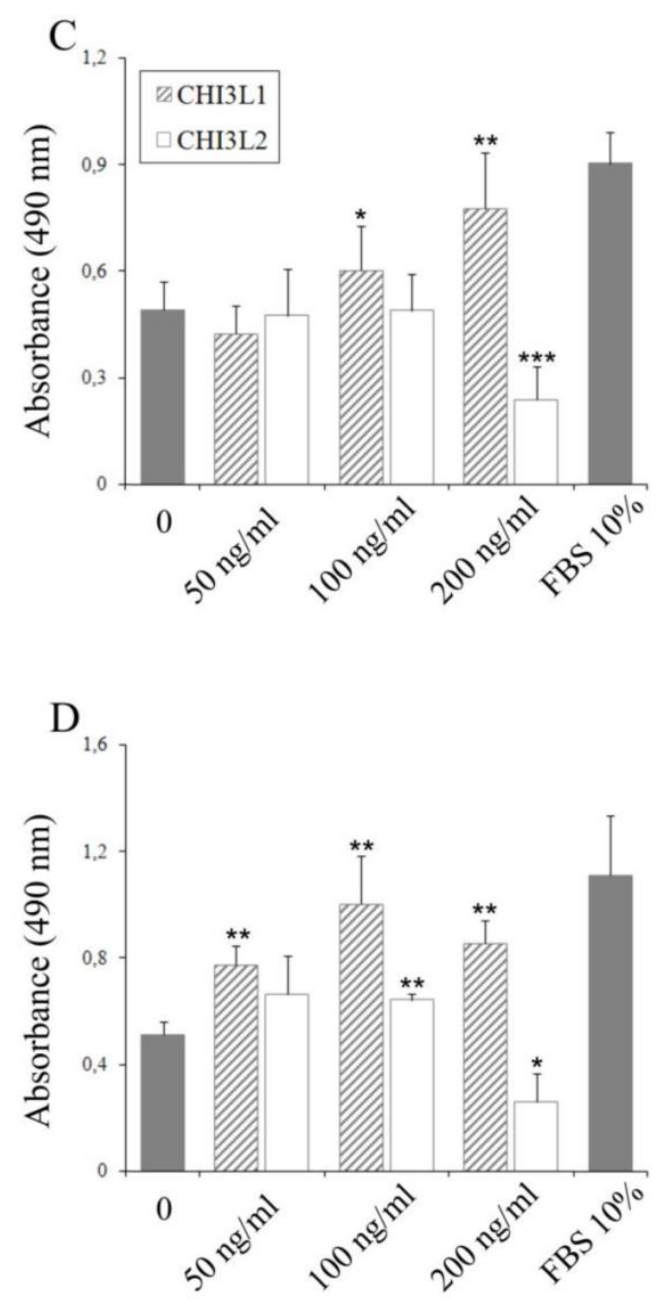

Figure I. Effect of CHI3LI and CHI3L2 on 293 cells and $\mathrm{U} 373$ cells growth. Dose-dependent effect of CHI3LI and CHI3L2 application on $\left[{ }^{3} \mathrm{H}\right]$ thymidine incorporation in 293 cells $(A)$ and U373 cells (B). Dose-dependent effect of CHI3LI and CHI3L2 on 293 cells (C) and U373 cells (D) proliferation. $* p<0.05$, ${ }^{* *} p<0.01$ and $* * * p<0.00$ I vs control group. Values were means \pm SD $(n=6)$. 

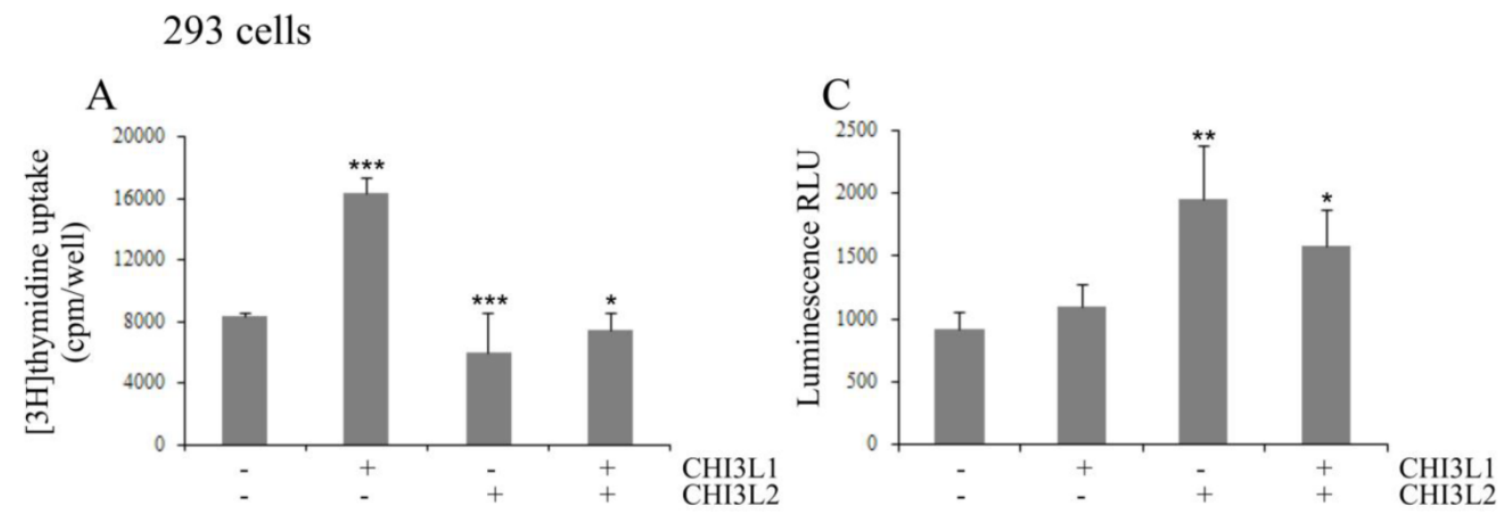

U373 cells
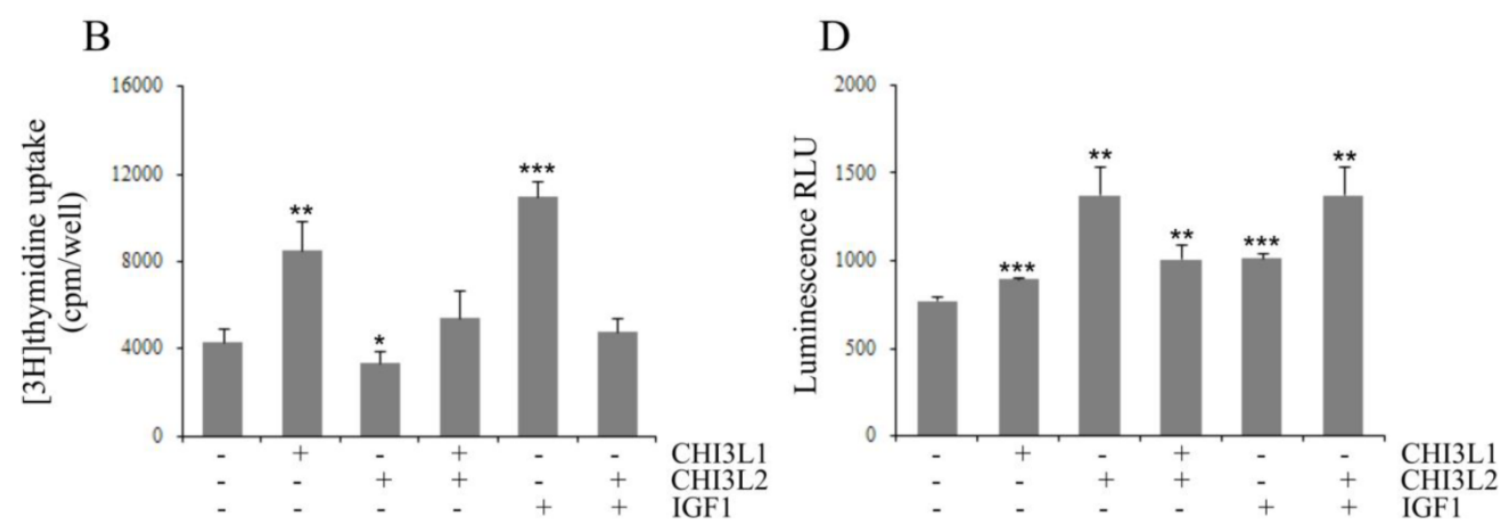

Figure 2. CHI3L2-inhibition of CHI3LI proliferative effect and CHI3LI-inhibition of CHI3L2-inducible apoptosis in 293 cells and U373 cells. Cultured 293 cells (A, C) or U373 cells (B, D) were untreated $(0 \mathrm{ng} / \mathrm{ml})$, treated for 24 hours with CHI3LI, CHI3L2, or IGFI (concentrations indicated) and treated with a mixture of these proteins. (A, B) DNA synthesis rate analyzed by $[3 \mathrm{H}]$ thymidine incorporation evaluation. (C, D) Caspase 3/7 activity determined by a caspase 3/7-Glo assay. ${ }^{*} p<0.05$, ${ }^{* *} p<0.01$ and ${ }^{* * *} p<0.001$ vs control group. Values were means \pm SD $(n=6)$.

\section{Distinguish in time course of CHI3LI- or CHI3L2-induced ERK I/2 phosphorylation and cellular localization of pERKI/2 in 293 cells and U373 cells}

The mitogen-activated protein kinase pathway is strongly associated with cell proliferation and survival. In this investigation to examine whether CHI3L2 can stimulate the MAP kinase pathway similarly to the closely related CHI3L1 protein we used human glioblastoma U373 cells. Same as with 293 cells [16], the obtained results indicate that addition of CHI3L1 or CHI3L2 stimulated ERK1/2phosphorylation in U373 cells in a dose-dependent manner (Fig. 3A). These cells, similarly to 293 cells, but in contrast to other glioblastoma cell line U87 [16] produce neither CHI3L1 nor CHI3L2 protein (Fig. 3B). No ERK1/2 phosphorylation was observed in cells exposed to medium without CHI3L1 or CHI3L2 addition. The phosphorylation of ERK1/2 and cell pro- liferation were inhibited by the MAP kinase kinase (MEK) inhibitor U0126, as expected (Fig. 4A, B) suggesting that MAP kinase-dependent signaling events modulate the cell response on CHI3L1 or CHI3L2 treatment.

In 293 cells, activation of ERK1/2 by CHI3L2 was more sustained (reaching a maximum after $60 \mathrm{~min}$ ) than by CHI3L1 (reaching a maximum after $20 \mathrm{~min}$ ), and declined after 2 hrs only by $\sim 30 \%$ while after activation by CHI3L1 it declined approximately to basal level (Figs. 5A, B). We were therefore interested to determine whether the difference in kinetics of ERK1/2 activation by CHI3L1 and CHI3L2 was accompanied by differences in phosphorylated ERK1/2 nuclear translocation. Results indicated that unstimulated 293 cells did not contain nuclear pERK1/2. After 5 min of stimulation by CHI3L1 nuclei (Fig. 5C) became brightly fluorescent, and even more so after stimulation by CHI3L2 (Fig. 5D). 
A

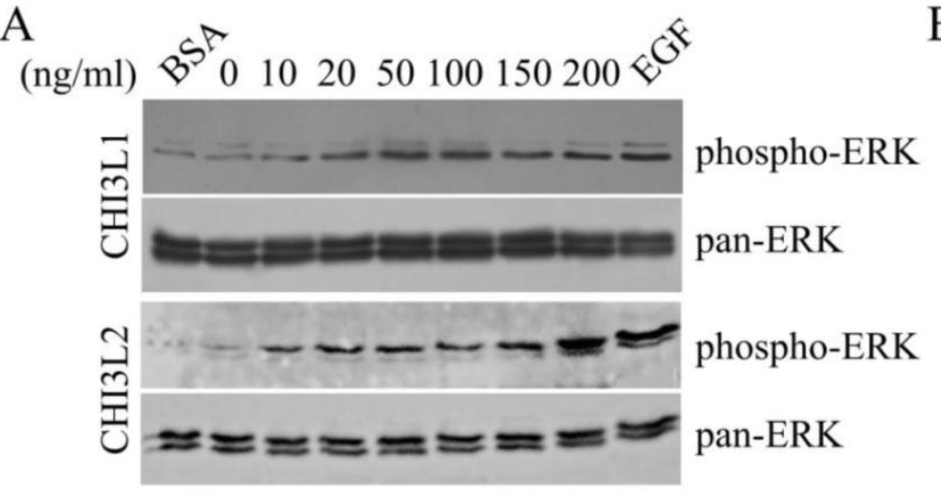

B

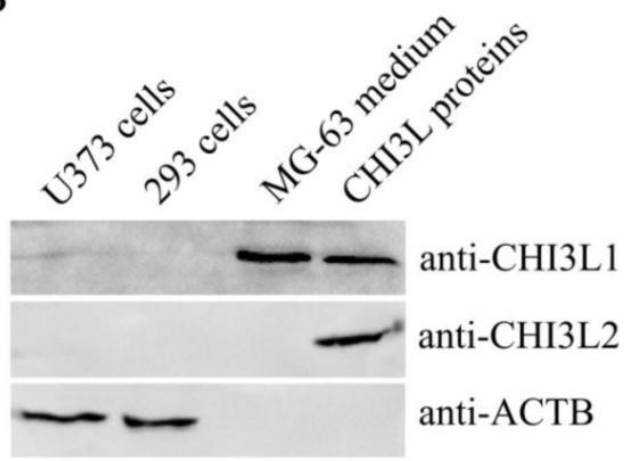

Figure 3. Dose-dependent phosphorylation of ERKI/2 induced by CHI3LI and CHI3L2. (A) Western-blot analysis of $U 373$ cells with anti-phospho-ERK and pan-anti-ERK antibodies after CHI3LI or CHI3L2 cell treatment (60 min). (B) Western-blot analysis of U373 cell, 293 cell, and MG-63 cell media or purified CHI3LI and CHI3L2 proteins with anti-CHI3LI, -CHI3L2 or -ACTB antibodies.

A

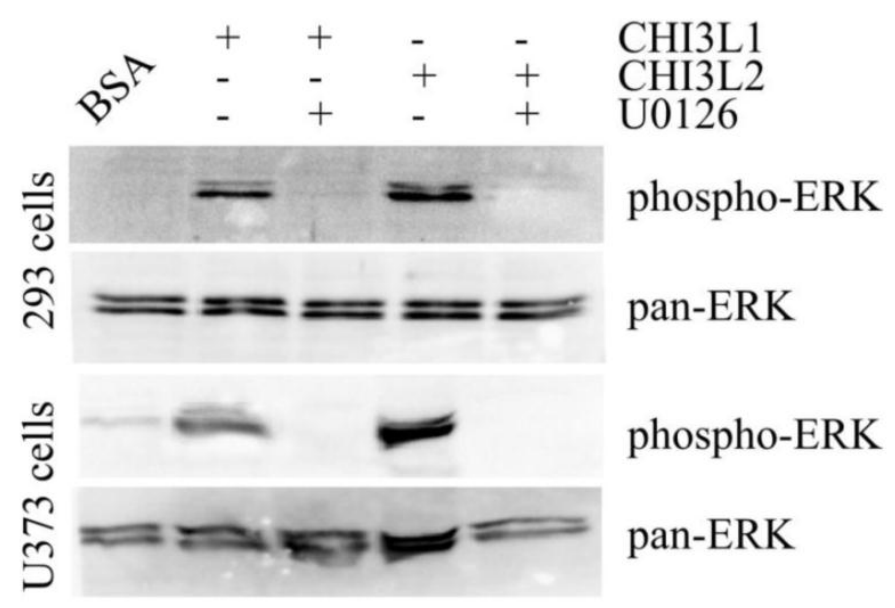

$\mathrm{B}$

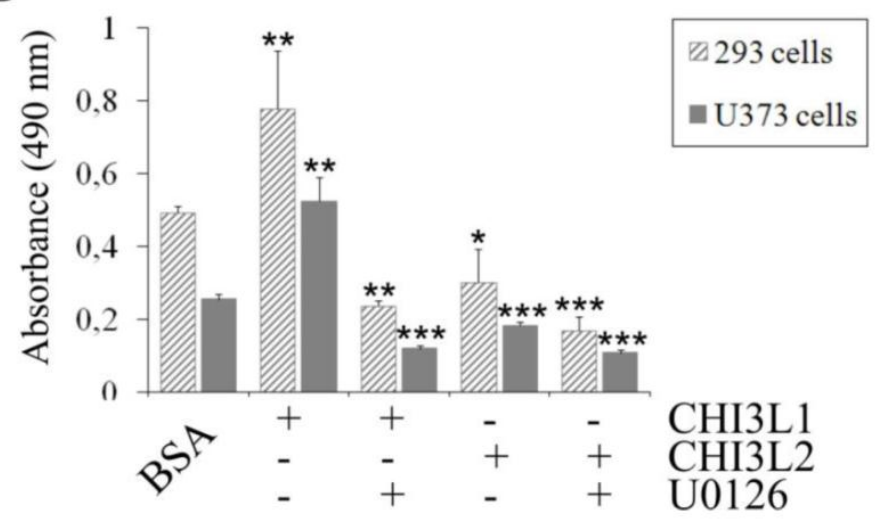

Figure 4. Influence of UOI 26 inhibitor on activation of ERKI/2-mediated MAPK signaling pathway. (A) 293 cells and U373 cells were serum-starved and exposed or not exposed to the ERKI/2 inhibitor U0I26 (20 $\mu \mathrm{M})$ for 30 min prior to addition of CHI3LI or CHI3L2 $(100 \mathrm{ng} / \mathrm{ml})$. Cell lysates were prepared after $60 \mathrm{~min}$ of stimulation and levels of phosphorylated and total ERK I/2 were analyzed as described for Figure 3 A. (B) Cell proliferation rate of 293 cells and U373 cells exposed or not exposed to the U0I 26 inhibitor after CHI3LI or CHI3L2 $(200 \mathrm{ng} / \mathrm{ml})$ stimulation $(24 \mathrm{hrs})$. ${ }^{*} p<0.05$, **p $<0.01$. Values were means \pm SD $(n=6)$. 


\section{3 cells}
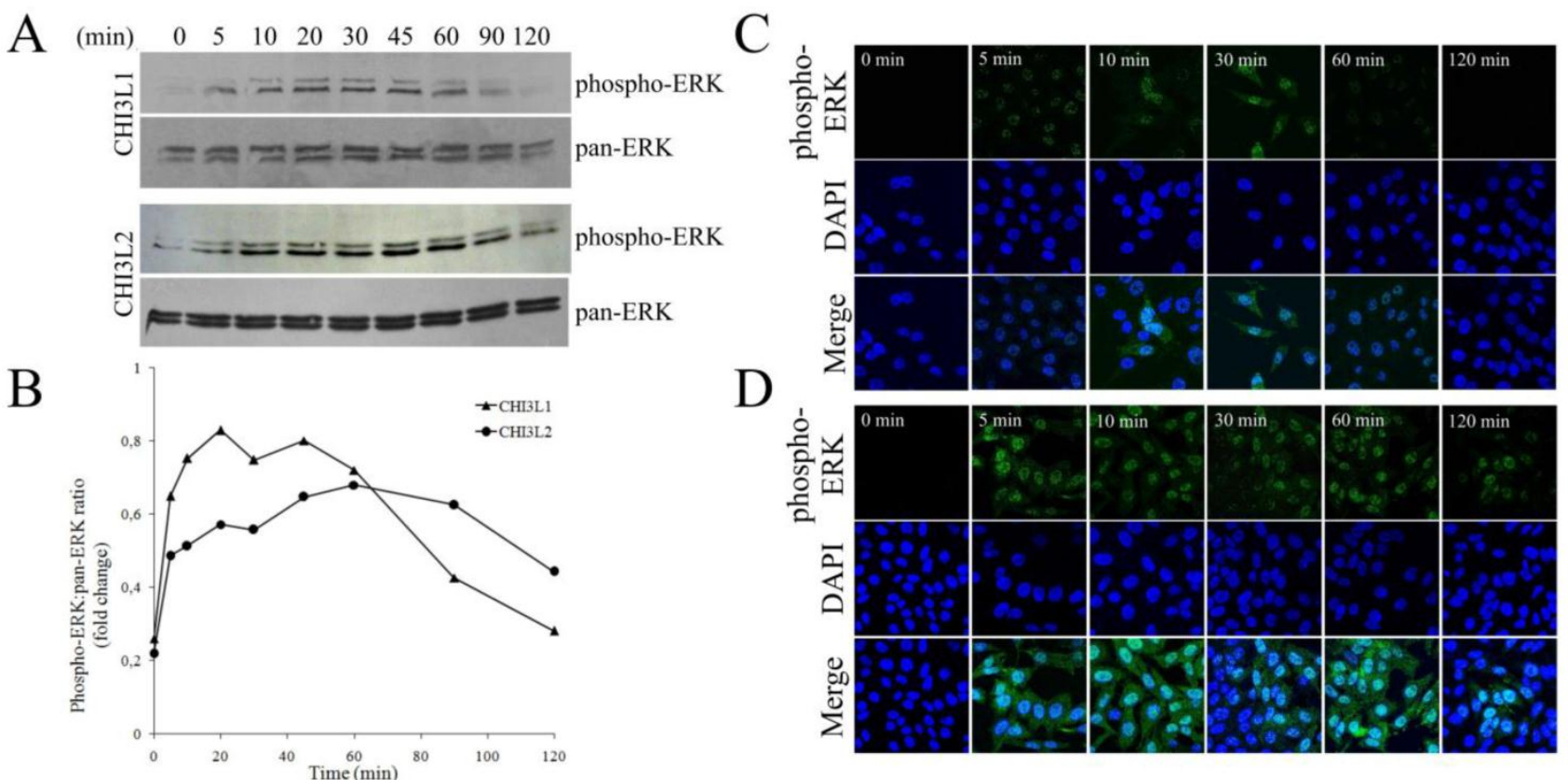

\section{U373 cells}
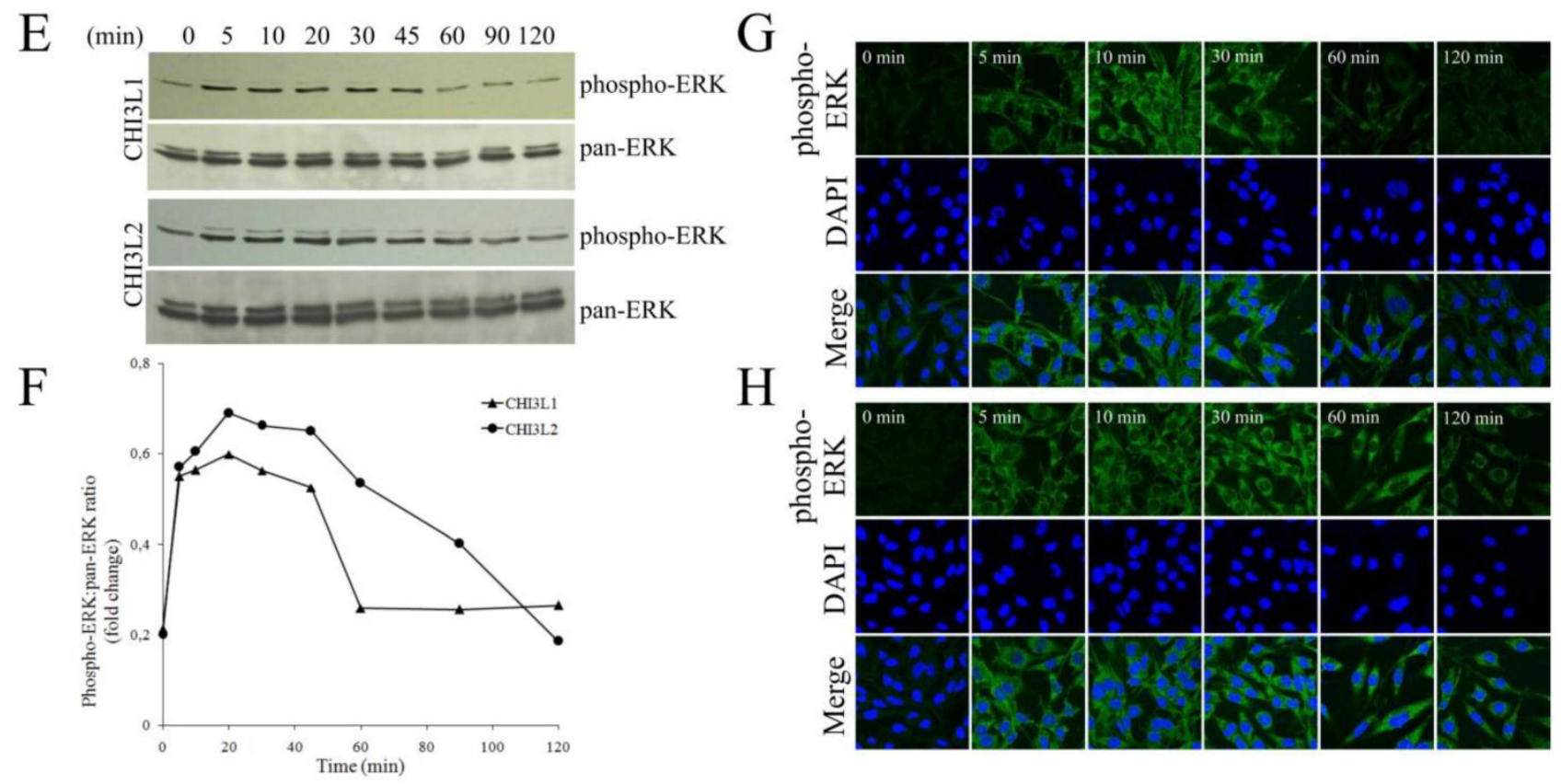

Figure 5. Time course of CHI3LI - or CHI3L2-induced ERKI/2 phosphorylation and nuclear accumulation in 293 cells and $U 373$ cells. (A,E), Time course of ERKI/2 phosphorylation by CHI3LI or CHI3L2 $(I 00 \mathrm{ng} / \mathrm{ml})$ treatment in 293 cells(A) or in U373 cells (E) . (B, F) Densitometric analysis of Figure A (B) or Figure E (F). Results are expressed as fold of phosphorylation of ERKI/2 compared with total ERKI/2. (C, G) Phosphorylated ERKI/2 cellular localization invoked by CHI3LI in 293 cells (C) or in U373 cells (G). (D,H) Phosphorylated ERKI/2 cellular localization invoked by CHI3L2 in 293 cells $(D)$ or in U373 cells $(H)$. 


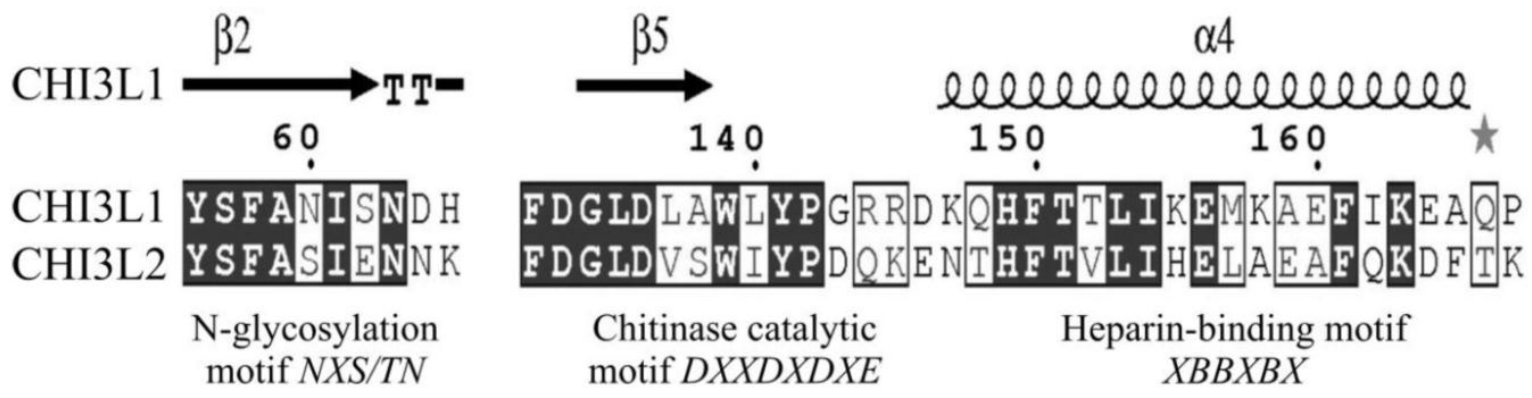

Figure 6. Key functional motifs of human CHI3LI and CHI3L2. Pairwise sequence alignment was produced with ESPript 2.2. Elements of secondary structure of CHI3LI are shown. B - basic amino acid, $\mathrm{X}$ - any, but proline.

In contrast to incubation with CHI3L1 that resulted in the maintenance of the pERK1/2 nuclear localization no longer than $30 \mathrm{~min}$ (Fig. 5C), nuclear fluorescence peaked at $10 \mathrm{~min}$ after CHI3L2 stimulation and was maintained for more than $2 \mathrm{hrs}$ (Fig. 5D).

In U373 cells, the observed ERK1/2 activation by CHI3L1 and CHI3L2 was quite similar to 293 cells, peaking in $20 \mathrm{~min}$ and declining to basal level in 60 min after CHI3L1 or in 120 min after CHI3L2 treatment (Fig. 5E, F). However, in contrast to 293 cells, localization of activated ERK1/2 was found only in the cytoplasm of U373 cells without nuclear translocation (Figs. 5G, H). So, CHI3L1 and CHI3L2 have not only different effects on the kinetics of ERK1/2 phosphorylation, but have also different effects on cellular localization of these enzymes in 293 cells compared to U373 cells.

\section{Difference of CHI3LI and CHI3L2 structure may result in antagonistic protein properties}

What is the mechanism(s) whereby CHI3L1, which activating ERK1/2 phosphorylation, leads to the mitogenesis and proliferation, and has oncogenic properties whereas $\mathrm{CHI}$ L2, which also activates ERK1/2 phosphorylation, leads to the decrease of mitogenesis and proliferation and may lead to differentiation? CHI3L1 and CHI3L2 belong to the chitinase-like family of proteins, but lack chitinase activity due to the mutation in the catalytic centre. These proteins are quite closely related (51\% homology), have same $\mathrm{NH}_{2}$-terminal aminoacid residues, and their 3-dimensional structures are also very similar [18].

The diversity in their functional activities could be explained, firstly, by the fact that native CHI3L1 is glycosylated at $\mathrm{Asn}^{60}$ while CHI3L2 is not a glycoprotein. Besides, CHI3L1 has a cluster of basic residues which can bind heparin; CHI3L2 has a different amino acid sequence in this site. Third, in the lig- and-binding groove CHI3L1 has two tryptophan residues, in CHI3L2 these tryptophans are mutated to lysines which change the protein charge and hydrophobicity (Fig. 6). Insights into the relationships between these features and tumorigenicity require further investigation.

\section{Discussion}

Although the investigation of CHI3L1 was mainly focused on its expression patterns in a number of pathological conditions [19], CHI3L2 the most closely related protein to CHI3L1 in the family 18 glycosyl hydrolases, is very poorly characterized. The growth-stimulating properties of one member of mammalian CLPs [20] may lead to the suggestion about conservation of this function throughout all members of the family. The results presented by Recklies et al. [13] indicate that CHI3L1 is a strong inductor of MAPK and PI3K signaling pathways in human connective tissue cells, mediating its action through the phosphorylation of ERK1/2 and AKT, respectively. Their finding fits with the highly investigated roles of these signaling pathways in the transduction of mitogenic signals [21-23].

In this study, ERK1 and ERK2 phosphorylation in 293 cells and U373 cells as well as in U87 cells [16] was induced by both CHI3L1 and CHI3L2. However, activation of ERK-mediated cellular signaling cascade can be associated with stimulation of mitogenesis and proliferation as in the case of CHI3L1 or inhibition of mitogenesis and proliferation as in the case of CHI3L2. PC12 cells have been extensively used as a model for study of how different receptor induction of ERK1/2 phosphorylation determines mitogenesis and proliferation or inhibition of mitogenesis and differentiation. Insights from these works suggest that cells make decisions about proliferation or differentiation using different duration of ERK1/2 activation and their localization: sustained ERK1/2 phosphorylation 
is associated with ERKs translocation to the nucleus while transient is not accompanied by nuclear translocation [24]. Transcription of genes important for differentiation or cell-cycle regulation, as well as stabilization of proteins, such as immediate early gene products, is regulated by duration and localization of ERK1/2 activity. Thus, the qualitative and quantitative differences in ERK1/2 activity lead to changes in gene expression that determined specific outcomes.

However, as it was discussed by Marshall [25], this model is based wholly on experiments on PC12 cells which may represent a very abnormal system. In our investigation, we observed that sustained ERK1/2 activation by CHI3L2 stimulated the translocation of these kinases into the cell nuclei, whereas transient ERK1/2 activation by CHI3L1 did not demonstrate such translocation similarly to the effects, described previously for NGF and EGF, correspondingly, in PC12 cells. Thus, we obtained the same effect with another cell type, namely, human 293 cells, where transient or sustained ERK1/2 activation leads either to stimulation or to inhibition of mitogenesis, respectively, and strongly supports the paradigm described for PC12 cells.

However, in fibroblasts sustained activation of ERKs was not associated with differentiation [26]. In glioblastoma-derived U373 cells (same as in the case of glioblastoma-derived U87 cells [16]) the phosphorylation of ERK1/2 was observed even without growth factors CHI3L1 or CHI3L2 addition, but reached a maximum after $20 \mathrm{~min}$ and declined after $2 \mathrm{hrs}$ approximately to basal level after either CHI3L1 or CHI3L2 application.

Activation of the ERK1/2 pathway is a normal response of the cell following stimulation of growth factors receptors by proper ligands. However, the identity of cellular receptors mediating the biological effects of CHI3L1 and CHI3L2 are currently not known, but the activation of cytoplasmic signal-transduction pathway suggests that these CLP's interacts with signaling components on the plasma membrane. ERK1/2-mediated pathway is shown to be inadequately activated in a variety of tumor cell lines and tumor tissue as it was shown here for the glioblastoma U373 cell line. In these cells all of the activated ERK1/2 remains in the cytoplasm without cytoplasm-to-nucleus translocation even after $2 \mathrm{hrs}$ of cell stimulation. Transient activation will therefore have very different consequences for gene expression compared with sustained activation in the cells of different origin because nuclear accumulation of active ERKs will result in phosphorylation of different transcription factors. Determination of the cellular response depending on previous developmental events that determine which ERK-responsive transcription factors are present in the 293 cells and U373 cells is the purpose of further investigations.

\section{Acknowledgments}

We thank Dr. S. Karakhim for providing expertise and access to Zeiss confocal microscope, Dr. I. Skrypkina for help in immunofluorescence analysis. This work was supported in part by Science \& Technology Center in Ukraine (Project 4688) and by National Academy of Sciences of Ukraine in frames of the program "Fundamentals of Molecular and Cellular Biotechnology".

\section{Abbreviations}

ACTB, $\beta$-actin; AKT, protein kinase B; BSA, bovine serum albumin; CHI3L1, chitinase 3-like 1; CHI3L2, chitinase 3-like 2; CLP, chitinase-like protein; DMEM, Dulbecco's modified Eagle's medium; EGF, epidermal growth factor; ERK, extracellular signal-regulated kinase; FBS, fetal bovine serum; FGF, fibroblast growth factor; IGF, insulin-like growth factor; MAPK, mitogen-activated protein kinase; NGF, nerve growth factor; PAGE, polyacrylamide gel electrophoresis; PC12, rat adrenal pheochromocytoma cell line; PBS, phosphate buffered saline; SDS, sodium dodecyl sulphate; TBS, Tris buffered saline.

\section{Conflict of Interests}

The authors have declared that no conflict of interest exists.

\section{References}

1. Raman M, Chen W, Cobb MH. Differential regulation and properties of MAPKs. Oncogene 2007; 26: 3100-12.

2. Traverse S, Gomez N, Paterson $\mathrm{H}$, et al. Sustained activation of the mitogen-activated protein (MAP) kinase cascade may be required for differentiation of PC12 cells. Comparison of the effects of nerve growth factor and epidermal growth factor. Biochem J. 1992; 288: 351-55.

3. Murphy LO, Smith S, Chen RH, et al. Molecular interpretation of ERK signal duration by immediate early gene products. Nat Cell Biol. 2002; 4: 556-64.

4. Henrissat B, Davies G. Structural and sequence-based classification of glycoside hydrolases. Curr Opin Struct Biol. 1997; 7: 637-44.

5. Bussink AP, Speijer D, Aerts JM, et al. Evolution of mammalian chitinase(-like) members of family 18 glycosyl hydrolases. Genetics. 2007; 177: 959-70.

6. Fusetti F, Pijning T, Kalk KH, et al. Crystal structure and carbohydrate-binding properties of the human cartilage glycoprotein-39. J Biol Chem. 2003; 278: 37753-60.

7. Garifulin OM, Shostak KO, Dmitrenko VV, et al. Increased expression of SOX-2 and HC gp-39 genes in astrocytic tumours. Biopol and Cell. 2002; 18: 324-29.

8. Tanwar MK, Gilbert MR, Holland EC. Gene expression microarray analysis reveals YKL-40 to be a potential serum marker for malignant character in human glioma. Cancer Res. 2003; 62: 4364-68. 
9. Shostak KO, Labunskyy VN, Dmitrenko VV, et al. HC gp-39 gene is upregulated in glioblastomas. Cancer Lett. 2003; 198: 203-10.

10. Saidi A, Javerzat $S$, Bellahcène $A$, et al. Experimental anti-angiogenesis causes upregulation of genes associated with poor survival in glioblastoma. Int J Cancer. 2008; 122: 2187-98.

11. Lee CG, Hartl D, Lee GR, et al. Role of breast regression protein 39 (BRP-39)/chitinase 3-like-1 in Th2 and IL-13-induced tissue responses and apoptosis. J Exp Med. 2009; 206: 1149-66.

12. Kavsan VM, Baklaushev NP, Balynska OV, et al. Gene encoding chitinase 3-like 1 protein (CHI3L1) is a putative oncogene. Intern J Biomed Sci. In press.

13. Recklies $\mathrm{AD}$, White $\mathrm{C}$, Ling $\mathrm{H}$. The chitinase 3-like protein human cartilage glycoprotein 39 (HC-gp39) stimulates proliferation of human connective-tissue cells and activates both extracellular signal-regulated kinase- and protein kinase B-mediated signalling pathways. Biochem J. 2002; 365: 119-26.

14. Hu B, Trinh $\mathrm{K}$, Figueira WF, et al. Isolation and sequence of a novel human chondrocyte protein to mammalian members of the chitinase protein family. J Biol Chem. 1996; 271: 19415-20.

15. Kavsan V, Dmitrenko V, Boyko O, et al. Overexpression of YKL-39 gene in glial brain tumors. Scholarly Research Exchange. 2008; doi: 10.3814/2008/814849.

16. Areshkov P, Kavsan V. Chitinase 3-like protein 2 (CHI3L2, YKL-39) activates phosphorylation of extracellular signal-regulated kinases ERK1/ERK2 in human embryonic kidney (HEK293) and human glioblastoma (U87 MG) cells. Tsitol Genet. 2010; 44: 3-9.

17. Harvey S, Weisman M, O'Dell J, et al. Chondrex: new marker of joint disease. Clin Chem. 1998; 44: 509-16.

18. Iershov A, Odynets K, Kornelyuk A, et al. Homology modeling of 3D structure of human chitinase-like protein CHI3L2 protein. Central Eur J Bio. 2010; 5: 407-20.

19. Johansen JS. Studies on serum CHI3L1 as a biomarker in diseases with inflammation, tissue remodelling, fibroses and cancer. Dan Med Bull. 2006; 53: 172-209.

20. De Ceuninck F, Gaufillier S, Bonnaud A, et al. YKL-40 (cartilage gp-39) induces proliferative events in cultured chondrocytes and synoviocytes and increases glycosaminoglycan synthesis in chondrocytes. Biochem Biophys Res Commun. 2001; 285: 926-31.

21. Moriguchi T, Gotoh Y, Nishida E. Roles of the MAP kinase cascade in vertebrates. Adv Pharmacol. 1996; 36: 121-37.

22. Waskiewicz AJ, Cooper JA. Mitogen and stress response pathways: MAP kinase cascades and phosphatase regulation in mammals and yeast. Curr Opin Cell Biol. 1995; 7: 798-805.

23. Datta SR, Brunet A, Greenberg ME. Cellular survival: a play in three akts. Genes Dev. 1999; 13: 2905-27.

24. Schramek H. MAP kinases: from intracellular signals to physiology and disease. News Physiol Sci. 2002; 17: 62-7.

25. Marshall CJ. Specificity of Receptor Tyrosine Kinase Signaling: Transient versus Sustained Extracellular Signal-Regulated Kinase Activation. Cell. 1995; 80: 179-85.

26. Pouysségur J, Volmat V, Lenormand P. Fidelity and spatio-temporal control in MAP kinase (ERKs) signalling. Biochem Pharmacol. 2002; 64: 755-63. 